\title{
Effect of Abdominal Breathing Combined with Brisk Walking on Intervention Effect of Female Patients with Essential Hypertension
}

\author{
Bing Zhao ${ }^{1} \&$ Haixia Wang ${ }^{1}$ \\ ${ }^{1}$ Hospital of Qingdao University of Science and Technology, QingDao, China \\ Correspondence: Haixia Wang, Hospital of Qingdao University of Science and Technology, QingDao 266061, \\ Shandong Province, China. Tel: 86-532-8895-6612
}

Received: December 18, 2020 Accepted: January 26, 2021 Online Published: February 5, 2021

doi:10.5539/gjhs.v13n3p76 URL: https://doi.org/10.5539/gjhs.v13n3p76

\begin{abstract}
Objective: Exploring the intervention effect of abdominal breathing combined with brisk walking on female patients with essential hypertension.

Methods: 98 female patients diagnosed as essential hypertension were randomly divided into simple walking group and abdominal breathing combined with walking group, and the intervention effects of the two groups were compared.
\end{abstract}

Results: Abdominal breathing combined with brisk walking was better than brisk walking alone in BMI, systolic blood pressure, total cholesterol, low density lipoprotein and other aspects $(\mathrm{P}<0.05)$.

Conclusion: Abdominal breathing combined with brisk walking exercise can improve the therapeutic effect of female patients with essential hypertension and improve their self-management ability for chronic diseases.

Keywords: chronic diseases, essential hypertension, abdominal breathing, brisk walking, therapeutic effect

\section{Introduction}

In recent years, with the improvement of living standards and the change of lifestyle, chronic non-communicable diseases have become the main disease burden in society (Hu, Rao, Qian, \& Wu, 2007). By the end of 2018, China's elderly population aged 60 and above is about 249 million, accounting for $17.9 \%$ of the total population, of which nearly 180 million suffer from chronic diseases, and the health of the elderly cannot be ignored (Healthy China Action Promotion Committee, 2020). Hypertension, as one of lifestyle diseases, has a incidence rate of over 270 million in our country and is the primary risk factor for cardiovascular diseases. It has the characteristics of high incidence rate, high disability rate and high mortality rate and seriously endangers the health of the elderly (Liang, Liu, Du, \& Qiu, 2014). Changing unhealthy lifestyle and regular and moderate aerobic exercise are helpful to prevent and improve chronic diseases such as hypertension, which has become the consensus of people (Gu, Weng, \& $\mathrm{Lu}, 2020$ ). How to use exercise prescriptions and medical resources to help patients with essential hypertension, improve the quality of life and reduce disability and mortality is particularly important (Li \& Huang, 2018). In this study, abdominal breathing combined with brisk walking was used to explore its intervention effect on female hypertension patients.

\section{Data and Methodology}

\subsection{Clinical Data}

Clinical data were selected from 98 female patients with essential hypertension diagnosed from January 2019 to June 2019, aged 55-65 years, with an average age of $60.11 \pm 2.13$. They were randomly divided into 2 groups by random number table: simple walking group (group A, 40 cases) and abdominal breathing combined with walking group (group B, 58 cases). There was no significant difference between the two groups in baseline data $(\mathrm{P}>0.05)$. Selection criteria: (1) After 3 non-day blood pressure tests, systolic blood pressure increased $\geq 140$ $\mathrm{mmHg}$ and diastolic blood pressure increased $\geq 90 \mathrm{mmHg}$ (one of the two conditions is met), and the reason is unknown; (2) It meets the standard of simple hypertension and has no complications. The hypertension grade is Grade 1 or Grade 2. (3) Clear consciousness and normal cognitive function; (4) Able to master the communication of social tools such as telephone, WeChat and QQ. Exclusion criteria:(1) Diagnosis of heart, brain, kidney and other important organ diseases or hypertension is above grade 3; (2) There is injury to the motor system. (3) Participation in other sports except those required by the experiment. 


\subsection{Exercise Intervention Methods}

All participants filled in PAR-Q questionnaire and formulated exercise prescription to ensure medical supervision. The exercise process includes 5-10 minutes of preparation activities, 30-40 minutes of sports and 5 minutes of finishing activities. Sports adopt the mode of brisk walking, the exercise intensity is $65-75 \%$ of maximum heart rate (220-age), and the exercise frequency is 3-5 times a week. The movement method is to hold your head high and hold your chest high. On the basis of natural walking, the upper limb swings back and forth with the shoulder joint as the movement axis, and the lower limb transitions from the heel to the forefoot when landing.

Group B took abdominal breathing for half an hour every morning on the basis of brisk walking. The method was to place both hands on the navel and breathe backwards. The abdomen was sunken when inhaling and bulged when exhaling, with a frequency of 8-9 times per minute.

The contents of individualized health management include (1) understanding the basic information of patients and obtaining their knowledge and consent; (2) Education on hypertension-related knowledge, including nutrition intake, sleep education, psychological care, physical exercise, etc. (3) Selection and application of hypertension drugs; (4) Exercise training, including effective abdominal breathing and brisk walking, breathing coordination during brisk walking, etc.

\subsection{Measurement Indicators}

The measurement indexes include morphological indexes such as height, weight, body mass index (BMI), waist circumference, hip circumference, etc. Functional indexes such as heart rate and blood pressure include systolic blood pressure (SBP) and diastolic blood pressure (DBP), etc. Blood indexes such as fasting blood glucose (FBG), triglyceride (TG), total cholesterol (CHOL), low density lipoprotein (LDL), high density lipoprotein (HDL) and so on.

\subsection{Statistical Method}

SPSS17.0 software was adopted in this paper, and the measurement data were mean $\pm \mathrm{SD}(\mathrm{P}<0.05$ was statistically significant). The comparison adopted independent sample $\mathrm{T}$ test or paired $\mathrm{T}$ test.

\section{Results}

\subsection{Changes of Data Before Exercise Intervention in the Two Groups of Patients}

Table 1. Comparison of data changes between the two groups before exercise intervention

\begin{tabular}{lcccc}
\hline & Group A (mean \pm SD $)$ & Group B (mean \pm SD $)$ & T & -0.182 \\
\hline Age & $60.07 \pm 3.06$ & $60.17 \pm 2.24$ & -0.129 & 0.864 \\
BMI & $23.92 \pm 0.74$ & $23.94 \pm 0.74$ & 1.533 & 0.119 \\
Waistline & $84.53 \pm 6.31$ & $82.34 \pm 7.31$ & 0.618 & 0.539 \\
Hip circumference & $95.70 \pm 5.51$ & $95.02 \pm 5.18$ & -0.395 & 0.694 \\
Heart rate & $81.16 \pm 7.36$ & $81.78 \pm 81.78$ & -0.546 & 0.586 \\
SBP & $160.80 \pm 11.23$ & $159.53 \pm 11.31$ & 1.603 & 0.113 \\
DBP & $93.85 \pm 9.02$ & $91.16 \pm 6.77$ & -1.429 & 0.158 \\
FBG & $5.62 \pm 0.50$ & $5.75 \pm 0.34$ & 0.591 & 0.556 \\
TG & $1.41 \pm 0.49$ & $1.35 \pm 0.44$ & 0.082 & 0.935 \\
CHOL & $4.20 \pm 1.37$ & $4.18 \pm 1.37$ & -0.861 & 0.392 \\
HDL & $1.45 \pm 0.46$ & $1.53 \pm 0.50$ & 0.453 \\
LDL & $2.77 \pm 0.56$ & $2.72 \pm 0.57$ & 0.651 \\
\hline
\end{tabular}

As can be seen from Table 1, there is no difference between group A and group B's 55-65-year-old women's body data before exercise intervention at a level greater than 0.05 , showing homogeneity, and exercise intervention can be carried out. 


\subsection{Comparison of the Situation of Group a Patients after 16 Weeks}

Table 2. Comparison of data changes before and after exercise intervention in group A

\begin{tabular}{lcccc}
\hline & $\begin{array}{c}\text { Before exercise intervention } \\
\text { in group A (mean } \pm \text { SD) }\end{array}$ & $\begin{array}{c}\text { After exercise intervention } \\
\text { in group A (mean } \pm \text { SD) }\end{array}$ & T & P \\
\hline BMI & $23.92 \pm 0.74$ & $23.26 \pm 0.76$ & 5.932 & 0.000 \\
Waistline & $84.53 \pm 6.31$ & $80.65 \pm 5.12$ & 7.133 & 0.000 \\
Hip circumference & $95.70 \pm 5.51$ & $92.58 \pm 5.34$ & 13.281 & 0.000 \\
Heart rate & $81.16 \pm 7.36$ & $77.00 \pm 8.44$ & 2.887 & 0.006 \\
SBP & $160.80 \pm 11.23$ & $146.48 \pm 7.68$ & 12.110 & 0.000 \\
DBP & $93.85 \pm 9.02$ & $85.05 \pm 5.92$ & 8.667 & 0.000 \\
FBG & $5.62 \pm 0.50$ & $5.14 \pm 0.47$ & 9.525 & 0.000 \\
TG & $1.41 \pm 0.49$ & $1.17 \pm 0.36$ & 7.691 & 0.000 \\
CHOL & $4.20 \pm 1.37$ & $3.68 \pm 1.13$ & 5.787 & 0.000 \\
HDL & $1.45 \pm 0.46$ & $1.55 \pm 0.45$ & -2.035 & 0.049 \\
LDL & $2.77 \pm 0.56$ & $2.47 \pm 0.52$ & 3.225 & 0.003 \\
\hline
\end{tabular}

As can be seen from Table 2, morphological indexes such as BMI, waist circumference, hip circumference, etc. of group A patients before and after exercise intervention; Functional indexes such as heart rate, blood pressure, etc. Blood indexes such as blood sugar, triglyceride, total cholesterol, low density lipoprotein and high density lipoprotein are less than 0.05 . This result shows that there are significant differences in various indexes before and after walking exercise intervention. To sum up, brisk walking exercise has played a positive role in improving morphological, functional and blood indexes of women aged 55-65.

\subsection{Comparison of the Situation of Group B Patients after 16 Weeks}

Table 3. Comparison of data changes before and after exercise intervention in group B

\begin{tabular}{lcccc}
\hline & $\begin{array}{c}\text { Before exercise intervention } \\
\text { in group B (mean } \pm \text { SD) }\end{array}$ & $\begin{array}{c}\text { After exercise intervention } \\
\text { in group B (mean } \pm \text { SD) }\end{array}$ & T & P \\
\hline BMI & $23.94 \pm 0.74$ & $22.90 \pm 0.70$ & 11.287 & 0.000 \\
Waistline & $82.34 \pm 7.31$ & $79.43 \pm 4.09$ & 4.864 & 0.000 \\
Hip circumference & $95.02 \pm 5.18$ & $90.79 \pm 4.31$ & 4.431 & 0.000 \\
Heart rate & $81.78 \pm 81.78$ & $73.09 \pm 5.02$ & 10.337 & 0.000 \\
SBP & $159.53 \pm 11.31$ & $137.16 \pm 6.85$ & 18.994 & 0.000 \\
DBP & $91.16 \pm 6.77$ & $85.26 \pm 5.38$ & 9.449 & 0.000 \\
GLU & $5.75 \pm 0.34$ & $5.07 \pm 0.57$ & 7.808 & 0.000 \\
TG & $1.35 \pm 0.44$ & $1.16 \pm 0.31$ & 3.623 & 0.000 \\
CHOL & $4.18 \pm 1.37$ & $3.23 \pm 0.64$ & 5.205 & 0.000 \\
HDL & $1.53 \pm 0.50$ & $1.55 \pm 0.48$ & -3.543 & 0.001 \\
LDL & $2.72 \pm 0.57$ & $2.05 \pm 0.76$ & 4.699 & 0.000 \\
\hline
\end{tabular}

As can be seen from Table 3, morphological indexes such as BMI, waist circumference, hip circumference, etc. of group B patients before and after exercise intervention; Functional indexes such as heart rate, blood pressure, etc. Blood indexes such as blood sugar, triglyceride, total cholesterol, low density lipoprotein and high density lipoprotein are less than 0.05 . This result shows that there are significant differences in various indexes before and after walking exercise intervention. To sum up, abdominal breathing combined with brisk walking exercise has played a positive role in improving morphological, functional and blood indexes of women aged 55-65. 


\subsection{Comparison of the Situation between Group A and Group B after16 Weeks of Exercise Intervention}

Table 4. Comparison of data changes between the two groups after exercise intervention

\begin{tabular}{lcccc}
\hline & $\begin{array}{c}\text { After exercise intervention } \\
\text { in group A (mean } \pm \text { SD) }\end{array}$ & $\begin{array}{c}\text { After exercise intervention in } \\
\text { group B (mean } \pm \text { SD) }\end{array}$ & T & P \\
\hline BMI & $23.26 \pm 0.76$ & $22.90 \pm 0.70$ & 2.424 & 0.018 \\
Waistline & $80.65 \pm 5.12$ & $79.43 \pm 4.09$ & 1.256 & 0.213 \\
Hip circumference & $92.58 \pm 5.34$ & $90.79 \pm 4.31$ & 1.752 & 0.084 \\
Heart rate & $77.00 \pm 8.44$ & $73.09 \pm 5.02$ & 2.436 & 0.018 \\
SBP & $146.48 \pm 7.68$ & $137.16 \pm 6.85$ & 6.166 & 0.000 \\
DBP & $85.05 \pm 5.92$ & $85.26 \pm 5.38$ & -0.178 & 0.859 \\
FBG & $5.14 \pm 0.47$ & $5.07 \pm 0.57$ & 0.668 & 0.506 \\
TG & $1.17 \pm 0.36$ & $1.16 \pm 0.31$ & 0.034 & 0.973 \\
CHOL & $3.68 \pm 1.13$ & $3.23 \pm 0.64$ & 2.295 & 0.026 \\
HDL & $1.55 \pm 0.45$ & $1.55 \pm 0.48$ & -0.008 & 0.994 \\
LDL & $2.47 \pm 0.52$ & $2.05 \pm 0.76$ & 3.312 & 0.001 \\
\hline
\end{tabular}

As can be seen from Table 4, morphological indexes such as BMI; of patients in group A and group B after exercise intervention; Functional indexes such as heart rate, systolic blood pressure, etc. Blood indexes such as total cholesterol and low density lipoprotein are less than 0.05 . This result shows that there are significant differences in BMI, systolic blood pressure, total cholesterol and low density lipoprotein between group A and group B after exercise intervention. To sum up, abdominal breathing combined with brisk walking exercise has a better effect on improving some morphological, functional and blood indexes of women aged 55-65 years old than simple brisk walking exercise.

\section{Discussion}

Cardiovascular and cerebrovascular diseases are the leading cause of death in our country, bringing heavy burden to families and society. Hypertension, as one of the major and changeable risk factors for cardiovascular and cerebrovascular diseases, has a incidence rate of $25.2 \%$ among Chinese adults (Liu, 2010). Because the etiology of $95 \%$ of hypertension patients is unknown, it is called essential hypertension. $50 \%$ of essential hypertension is complicated with other high-risk factors of cardiovascular and cerebrovascular diseases. Therefore, how to better control blood pressure is the goal that doctors and patients have been striving for. Healthy lifestyle can prevent or delay the occurrence of hypertension, and improving lifestyle has been listed as the first-line antihypertensive method (Writing Group of 2018 Chinese Guidelines for the Management of Hypertension, 2019), of which moderate intensity aerobic exercise of more than 150 minutes per week is beneficial to prevent or treat hypertension (CPC Central Committee and State Council, 2016).

Aerobic exercise as the main form of exercise can reduce sympathetic nerve excitability, reduce the activity of blood-boosting hormones in the body, reduce the peripheral resistance of blood vessels, and remove cholesterol and other substances in the body, thus playing a role in lowering blood pressure (Liu, Simao, , Ding, \& Quansheng, 2008). As one of the simple and easy aerobic exercise methods, brisk walking exercise was an exercise method of invigorating qi and keeping fit in ancient times. Because it is easy to master and adhere to, is not limited by venues and time, and causes little sports damage to athletes, it should be popularized among the middle-aged and elderly people (Yan et al., 2017). In this study, we have trained all participants in walking, the intensity, duration and frequency of brisk walking were stipulated. It was found that in the two groups of personnel, after 12 weeks of exercise intervention, blood pressure improved to different degrees, including systolic blood pressure and diastolic blood pressure decreased, which proved that regular and regular aerobic exercise can reduce blood pressure. In the study, it was also found that brisk walking exercise has a good effect on controlling weight, waist circumference and hip circumference, and overweight or obesity is one of the risk factors for hypertension. The study also found that the patient's heart rate decreased after regular brisk walking exercise. Because the change of heart rate has a relatively large influence on diastolic blood pressure, when the heart rate slows down, the diastolic period of the heart becomes longer, thus increasing the amount of blood flowing to the periphery and reducing diastolic blood pressure, so as to reduce functional or organic changes of organs such as heart, brain, kidney and retina. The study also found that brisk walking also plays a role in 
reducing blood sugar and blood lipid, and hyperglycemia and hyperlipidemia are also one of the risk factors for cardiovascular and cerebrovascular diseases, especially the increase of total cholesterol and low density lipoprotein.

Abdominal breathing refers to the breathing mode dominated by diaphragm movement. Abdominal breathing is the main mode in adult men and children, while chest breathing is the main mode in adult women. Because chest respiration is shallow, it is easy to cause hypoxia in various organs of the body, while abdominal respiration can fully expand organs above and below lungs and diaphragms, play a massage role and increase the activity of parasympathetic nerve, so it is highly respected by ancient people and is believed to have the effect of prolonging life. In this study, On the basis of instructing women to walk briskly, Half an hour of abdominal breathing every day, Compared with women who only walk in brisk steps, systolic blood pressure is obviously decreased, while diastolic blood pressure is not obviously decreased, suggesting that abdominal breathing combined with walking in brisk steps is more conducive to systolic blood pressure control, and is more obvious in heart rate control, total cholesterol and low density lipoprotein reduction, which is more conducive to reducing the risk of cardiovascular and cerebrovascular diseases.

Through the above research, we can draw the following conclusions: 1. Walking as aerobic exercise can improve and mediate the treatment of hypertension in female patients. 2. Abdominal breathing combined with brisk walking exercise can further improve the therapeutic effect of female patients with essential hypertension on the basis of brisk walking exercise and improve the self-management ability of patients for chronic diseases. Therefore, we suggest to strengthen the promotion of abdominal breathing, so as to arouse women's attention to abdominal breathing, and to promote the concept of national fitness life, to play the positive role of national scientific fitness in health promotion, chronic disease prevention and rehabilitation.

\section{Competing Interests Statement}

The authors declare that there are no competing or potential conflicts of interest.

\section{References}

CPC Central Committee and State Council. (2016). Outline of "Healthy China 2030" Plan. 2016-10-25

Gu, D. F., Weng, J. P., \& Lu, X. F. (2020). Chinese Guideline on Healthy Lifestyle to Prevent Cardiometabolic Diseases. Chinese Circulation Journal, 35(3), 209-230.

Healthy China Action Promotion Committee. (2020). Healthy China Action (2019-2030). http://www.nhc.gov.cn/wsxf/zcfg/201907/2a771d89e00e4b228028335d4fcfla7d.shtml.2020.10.223.

Hu, J. P., Rao, K. Q., Qian, J. C., \& Wu, J. (2007). The study of economic burden of chronic non-communicable diseases in China. Chinese Journal of Prevention and Control of Chronic Non Communicable Diseases, 15(3), 189.

Li, H. W., \& Huang, Z. Q. (2018). Experimental Study of Intelligent Exercise Prescription Intervention and Monitoring of Critical Hype Hypertension Based on "Combination of Sports and Medicine". Journal of Wuhan Institute of Physical Education, 52(8), 90-95.

Liang, Y., Liu, R., Du, S., \& Qiu, C. (2014). Trends in incidence of hypertension in Chinese adults, 1991-2009: the China Health and Nutrition Survey. International journal of cardiology, 175(1), 96-101. https://doi.org/10.1016/j.ijcard.2014.04.258

Liu, L. S. (2010). Chinese guidelines for the management of hypertension. Chin J Hypertens, 19(8), 701-742.

Liu, T., Simao, X. U., Ding, H., \& Quansheng, S. U. (2008). Effects of aerobic exercise on cardiovascular functions and blood indexes. Chinese Journal of Tissue Engineering Research, 12(15), 2965-2968.

Writing Group of 2018 Chinese Guidelines for the Management of Hypertension. (2019). Chinese guidelines for the management of hypertension. Chin J Cardiol, 24(1), 24-56

Yan, X. W., Yang, Y. J., Fu, J. J., et al. (2017). Study on effect of walking indicators intervention on occupational health population. Chinese Journal of Disease Control \& Prevention, 21(5), 534-536.

\section{Copyrights}

Copyright for this article is retained by the author(s), with first publication rights granted to the journal.

This is an open-access article distributed under the terms and conditions of the Creative Commons Attribution license (http://creativecommons.org/licenses/by/4.0/). 